\title{
Continuous Wavelet Analysis and Classification of Surface Electromyography Signals
}

\author{
J. Kilby and K. Prasad
}

\begin{abstract}
The purpose of this research is to classify Surface Electromyography (SEMG) signals for normal muscle activity. The aim is to use different extracted features from Continuous Wavelet Transform (CWT) to build and train an Artificial Neural Network (ANN). The extracted features of the SEMG signals used in this research are the mean frequency and the median frequency of the Fourier Power Spectrum along with the RMS values of the signal. These features will be extracted at the selected scales of 8, 16, 32, 64 and 128 of the CWT. The SEMG were collected from normal vastus lateralis and vastus medialis muscles of both legs from $\mathbf{4 5}$ male subjects at $\mathbf{2 5 \%}$, $50 \%$, and $75 \%$ of their Maximum Voluntary Isometric Contraction (MVIC) force of the quadriceps. Using CWT for extracting features in order to analyse and classify SEMG signals by an ANN has shown to be sound and successful for the basis implementation for developing an intelligent SEMG signal classifier.
\end{abstract}

Index Terms - Component, electromyography, wavelet, fourier, neural network.

\section{INTRODUCTION}

Feature extraction and pattern recognition is the key in processing and analysing bio-medical signals. The use of the signal analysis is apparent in the field of clinical health for diagnosing health related problems and rehabilitation using bio-medical signals such as Electromyography (EMG) signals. The EMG signals, also commonly known as myoelectric signals, are obtained by means of recording the activity of striated muscle using sensors or electrodes.

An accurate and computationally efficient means of classifying myoelectric signal patterns has been the subject of considerable research effort in recent years where having effective feature extraction is crucial for reliable classification [1]. Numerous research and studies concentrated on feature extraction and pattern recognition in the bio-medical signal or bio-signal processing have achieved tremendous contribution to the facilities developed and available for the signal analysis in the clinical field today.

In signal processing, determining the frequency content of a signal by Fourier transform is one of the main aspects in feature extraction and understanding the characteristics of a signal. However, obtaining the frequency content alone is not sufficient for analysing bio-medical signals due to it being non-stationary in nature [2], [3]. Fourier transform loses the time information after transforming time-based signal to frequency-based signal.

The Fourier transform of input signal $x(t)$ is defined as the

Manuscript received October 14, 2012; revised November 18, 2012.

The authors are with the School of Engineering at AUT University, Auckland, New Zealand (e-mail: jkilby@ aut.ac.nz; kprasad@laut.ac.nz). following notation as in (1) where $\omega$ is the angular frequency and $\omega=2 \pi f$ with $f$ is the input frequency. $x(t)$ is the time domain signal and $F(\omega)$ is its Fourier transform represented in frequency domain

$$
F(\omega)=\int_{-\infty}^{\infty} x(t) e^{-j \omega t} d t
$$

which is the sum over all time of the signal $x(t)$ multiplied by complex exponential [4].

Since a digital computer only works with discrete data, a technique called Discrete Fourier Transform (DFT) is used [5]. FFT is the practical application name used for the DFT that maps discrete-time sequences into discrete-frequency representation as in (2) where $x[n]$ is the input sequence, $F(k)$ is the DFT, $2 \pi k$ is the angular frequency of input sequence frequency $k$ and $N$ is the number of samples in both discrete-time and the discrete-frequency domains.

$$
F(k)=\sum_{n=0}^{N-1} x[n] e^{-j 2 \pi k t / N}
$$

The mean frequency (MDF) is the average of all frequencies from the power spectrum and can be expressed as in (3) where $P(\omega)$ is the power spectrum of the signal.

$$
\int_{0}^{M D F} P(\omega) d \omega=\int_{M D F}^{\infty} P(\omega) d \omega
$$

The median frequency (MNF) is that frequency having $50 \%$ of the distribution on each side from the power spectrum and is given in (4).

$$
M N F=\frac{\int_{0}^{\infty} \omega P(\omega) d \omega}{\int_{0}^{\infty} P(\omega) d \omega}
$$

The collection of the SEMG signals from the surface of the skin to the data being stored involves a number of different stages, starting from a continuous analogue signal and ending with a discrete or digital signal [6]. These stages include amplification, analogue-to-digital conversion and signal conditioning.

It is essential and in the interest of analysing bio-signal to obtain 'time-based' information of when a particular frequency content occurs [3], [7]. Wavelet transform is a method capable of achieving this which is so called the time-frequency content or the time-frequency based representation.

The mathematical expression of a wavelet family which consists of members or daughter wavelets, $\psi_{a, \tau}$ is obtained by 
scaling and time shifting of the mother wavelet $\psi(t)$ defined as in (5) where $a \in \mathfrak{R}^{+}$represents scale parameter and $\tau \in \mathfrak{R}$ represent the translation parameter. When $a$ becomes large, the basis function $\psi_{a, \tau}$ becomes a stretched version of the prototype, which emphasises the low-frequency components. A small $a$ contracts the basis function $\psi_{a, \tau}$ and stresses the high-frequency components. However, the shape of the basis function will always remain unchanged [8].

$$
\psi_{a, \tau}(t)=\frac{1}{\sqrt{a}} \psi\left(\frac{t-\tau}{a}\right)
$$

Since $\psi(t)$ can be implemented as a band pass filter whose centre frequency can change, at a given scale, the filter yields wider or narrower frequency-response changes depending on the centre's frequency. This time-scale expression has an equivalent time-frequency expression. Since wavelets are well localised around a non-zero frequency $f_{0}$, at a scale $a=1$ (i.e. the mother wavelet), there is an inversely proportional relationship between scale and frequency, given by $a=f_{0} / f$

Note that the factor $1 / \sqrt{a}$ in (2) is introduced to guarantee energy preservation, that is to normalise the wavelet so that it has unit energy [8].

Other aspects of a signal such as the mean and median frequency of the power spectrum and the (RMS) value of signal's electrical potential also play an important role to the whole task of features extraction for signal characterisation. The ultimate aim of this exercise is to develop a system with the ability for signal classification by features, a powerful and promising tool for diagnosing problems.

The application of wavelet transform in analysing biological signals has only become increasingly developed in the last fifteen years [3], [9]. The wavelet theory is a relatively recent mathematical development where its application is a potentially promising and exciting area of research. Its application to the analysis of EMG signals is even more recent [3].

SEMG uses surface electrodes placed on the skin overlying the muscle observed. The other common EMG uses needle electrodes penetrated into the muscle, thus signals obtained are focused on a particular muscle motor unit. Needle EMG is an invasive method which can cause stress to the patient involved [10], hence SEMG is a preferable method of gathering signals. Furthermore, SEMG obtains signals sourced from a group of muscle rather than one single muscle unit.

This research designed and developed appropriate practice-oriented methodology and descriptive procedures for SEMG signal acquisitions, feature extraction, classification and validation by Artificial Neural Network (ANN).

This research explored and demonstrated the ability and potential in achieving reliable means for the diagnostic equipment development of a SEMG signal analyser using comparative database.

Traditionally, practical methodologies for pattern classification are the statistical and the syntactic approaches [8], [11]-[13]. Much early research on EMG signals classification found many difficulties associated with the statistical method of instrumenting many channels of the signal. The learning or neural approach in ANN is the most recent established type of pattern classifier which is a matured form of adaptive linear elements in learning algorithms [8], [14]-[16]. The first ANN began to appear in the mid 1980s where it could be a powerful method for classifying EMG signals. It is the feature set or data itself that is crucial to the overall performance of the classifier [8], which comes down to the selecting of the signal features that produce the best performance in terms of accuracy and efficiency [17]. Hence, a substantial set of database with sufficient quantity and effective selected features, may improve the classification of the EMG signals. ANN's potential ability as a powerful tool is yet required to be investigated for the classification purposes in this research. Research by Englehart et al [17] showed an effective representation for classification using ANN.

\section{Methodology}

\section{A. Subjects}

Forty five healthy volunteers with no previous history of knee or severe musculoskeletal injury (35 males and 10 females, age 18-35 years) participated in this study. This study was approved by the Auckland University of Technology Ethics Committee (AUTEC) and was performed after each subject had given written consent.

\section{B. SEMG Electrode Placement}

The SEMG signals were obtained from the vastus lateralis and vastus medialis muscles of both legs. Before the data collection, the subject's legs were shaved around the area of the muscles being tested. It was gently abraded with skin preparation cleanser, then cleansed with a $70 \%$ alcoholic swab and left to dry before attaching adhesive Ag (99.9\%) electrodes Norotrode $20^{\mathrm{TM}}$ bipolar silver/silver chloride electrodes (Myotronics, UK) with $20 \mathrm{~mm}$ inter-electrode spacing. The electrode was placed according to the set of recommendations published by Surface Electromyography for Non-invasive Assessment of Muscle (SENIAM) [18] as seen in Fig. 1. The reference electrode $3 \mathrm{M}^{\mathrm{tm}}$ Red Dot silver/silver chloride monitoring electrode (3M, USA) was attached to an area with no muscle tissue below the knee.

\section{Experimental Setup}

After the completion of a general warm-up, the subject was seated on a Biodex System 3 Pro dynamometer (Biodex Medical, Shirley, USA) with the upright chair set at $110^{\circ}$ and one knee bent to $90^{\circ}$. The load cell lever arm was attached to the chair that measured voluntary isometric force of the quadriceps.

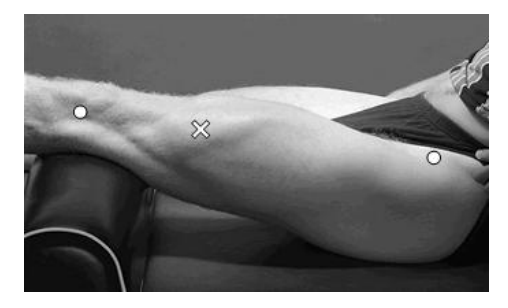

Fig. 1. Placement of electrode on the vastus lateralis muscle marked as a white cross between the two reference points marked as white dots.

Following the maximal strength tests, the subject performed a sustained force production test. This test was 
executed by having the subject push the load cell lever with $50 \%$ of MVIC force. The subject was required to perform and sustain the isometric contraction of the quadriceps at the given force level for a period of 10 seconds.

\section{Conventional Data Acquisition of SEMG Signals}

Signals from the SEMG electrodes were amplified at a gain of 100 and bandwidth filtered between $1 \mathrm{~Hz}$ and $3 \mathrm{kHz}$ using a Grass model P511 amplifier (Grass Instruments Company, USA). The analogue signals were acquired by a multifunction data acquisition board NI PCI-6024E (National Instruments Corporation, USA) with LabVIEW 2010 software (National Instruments Corporation, USA) for raw data acquisition on a host desktop computer. The signals were analogue-to-digital converted with 16 bit resolution in the $\pm 5 \mathrm{~V}$ range and sampled at $2048 \mathrm{~Hz}$.

\section{E. Signal Processing}

The recorded SEMG signals were subsequently analysed off-line using a newly developed virtual instrument (VI) within the software processing programme LabVIEW shown in Fig. 2. Any direct current (DC) component that may exist in the signals was removed before the analysis. The signals were subsequently digitally filtered using a $4^{\text {th }}$ order Butterworth band-pass filter with a pass-band from 5 to 500 $\mathrm{Hz}$.

For the feature extraction process, a section of the raw SEMG was selected. The selected region is an interval after the first peak activation as shown in Fig. 3. The selected first two seconds section (A) in Fig. 3 was not used for analysis. This region was to allow for changes in muscle tension at the beginning of the muscle contraction. The next two seconds section (B) in Fig.3 was the region to be used for analysis.

This region was where no muscle fatigue present and assumed to be quasi-stationary that is the stationary during short time intervals. Under this assumption spectral analysis for feature extraction can be applied $[19,20]$.

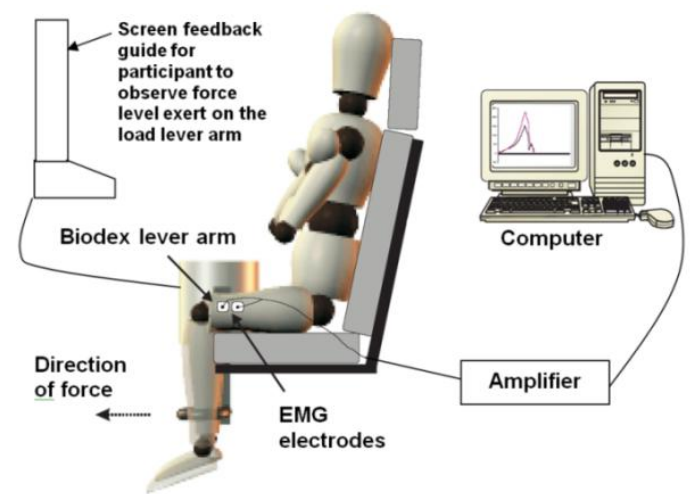

Fig. 2. Schematic diagram of equipment setup for signal acquisition.

Within this 2-second region, the initial scalogram was produced shown in middle plot in Fig. 4. The scalogram shows lower scale numbers as for the narrow version of the wavelet window which represent high frequencies, and the higher scale numbers for the wider windows represent as low frequencies. The conversion of scale index numbers to frequencies or pseudo frequencies were then carried out using the centre frequency $\mathrm{F}_{\mathrm{c}}$ of the mother wavelet, which in this case was the Morlet $\left(F_{c}=0.8125 \mathrm{~Hz}\right)$. A frequency-time based spectrum was plotted as a result of this conversion shown in the bottom plot of Fig. 4. A range of dominant frequencies could also be viewed from the frequency-time spectrum.

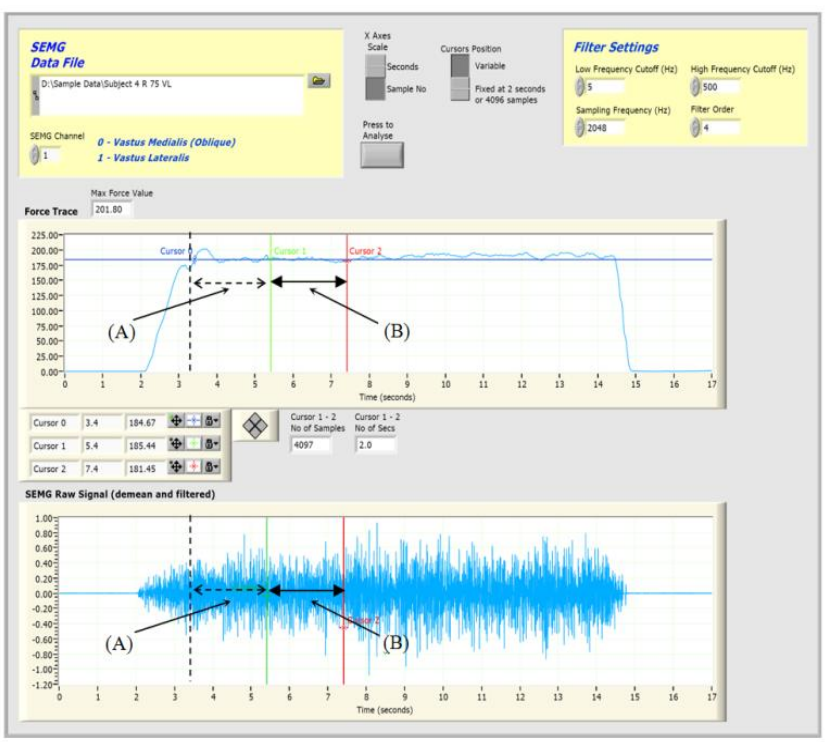

Fig. 3. Showing graph of force trace and raw SEMG signal displayed on a new developed LabVIEW VI. Part (A) first two second period to be ignored after first peak activation and Part (B) two period of muscle contraction to be analysed for feature extraction.

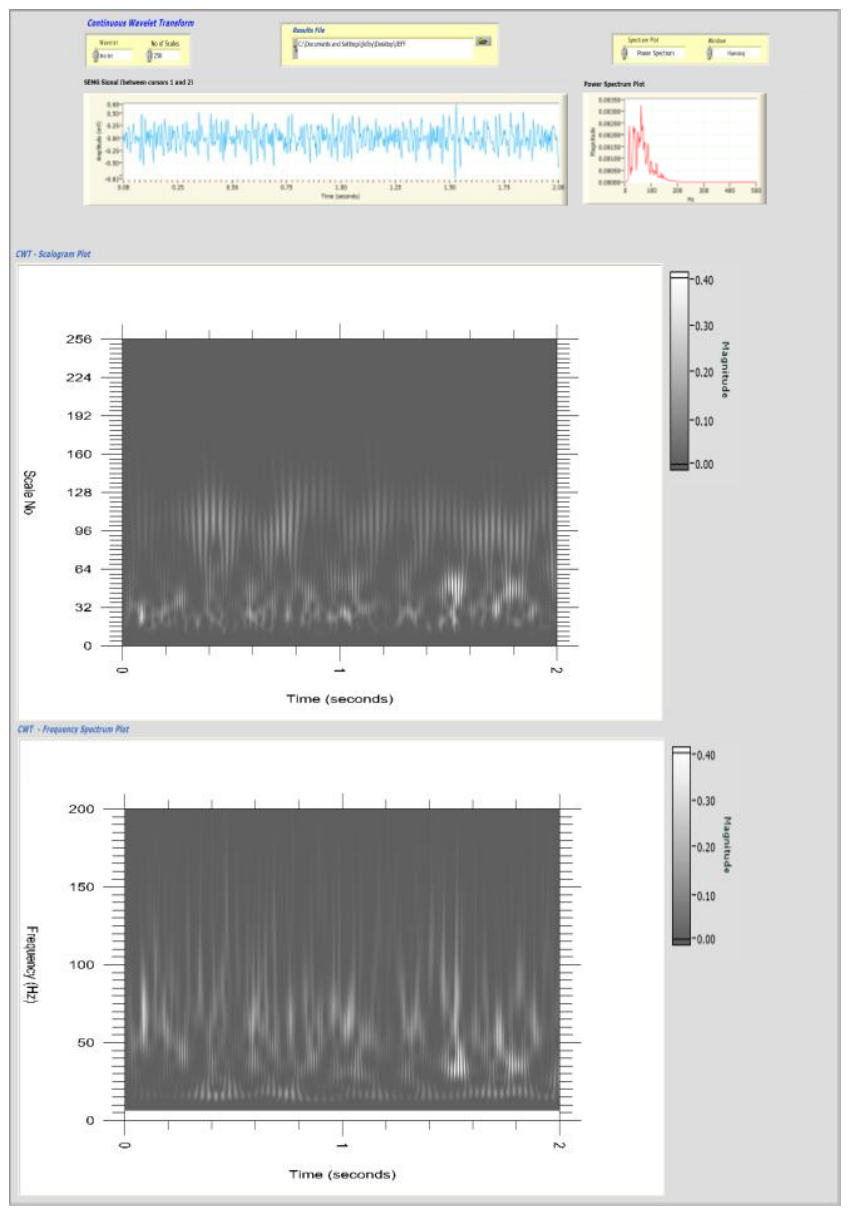

Fig. 4. The top plot shows the extracted two second period of the SEMG signal taken from the VI shown in Fig. 3. The middle plot shows CWT scalogram plot of scales against time. The bottom plot is corresponding CWT pseudo scalogram showing frequency against time. 

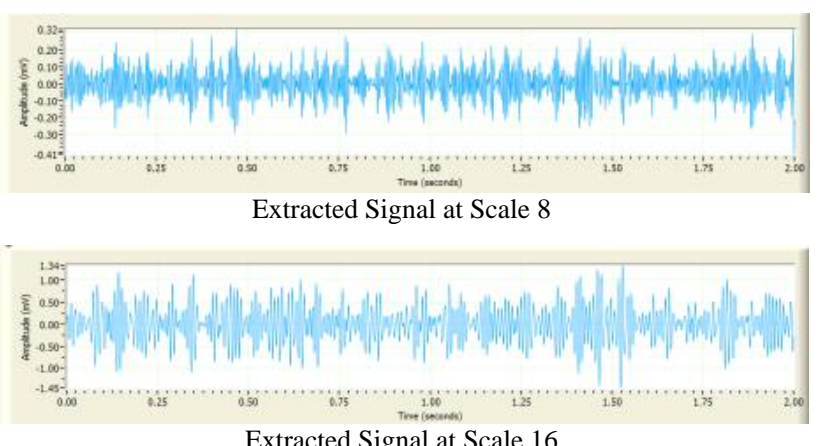

Extracted Signal at Scale 16

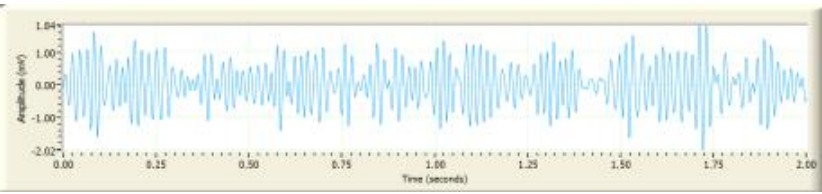

Extracted Signal at Scale 32



Extracted Signal at Scale 64

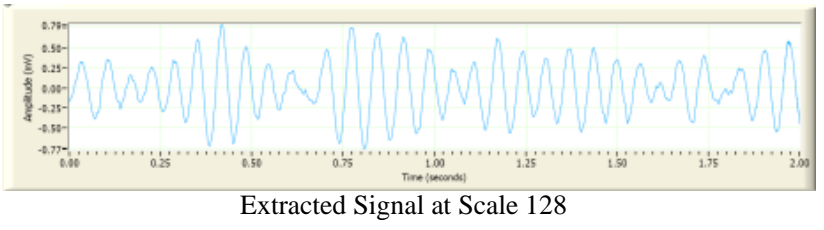

Fig. 5. Extracted signals at scales (a) 8, (b) 16, (c) 32, (d) 64 and (e) 128 from the scalogram plot shown in Fig. 4.

From the scalogram in Fig. 4, the scales selected were 8, 16, 32, 64 and 128. At each scale the transform wave was plotted in LabVIEW. These will then be analysed to extract the following features of MDF, MNF and RMS. These features are to be used to train and validate an ANN shown in Fig. 5.

\section{F. Artificial Neural Network}

The neural network architecture was built using MATLAB 2010 (MathWorks, USA) for this research and is illustrated in the Fig. 6. Fifteen input features from 35 subjects in the input layer were trained for 3 targets of 25\%, 50\% and 75\% MVIC in the output layer. In the hidden layer, the neuron numbers tested are from 3 to 10 to start with. The lower the number of neurons selected for the iterations, the faster the processing is.



Fig. 6. Neural Network Architecture build using MATLAB used for this research.
TABLE I: EXTRACTED FEATURES FROM THE RIGHT LEG'S VASTUS LATERALIS OF SUbJECT PERFORMING 75\% OF MVIC (SUBJECT No.4).

\begin{tabular}{|c|c|c|c|}
\hline Scales & $\begin{array}{c}\text { Mean } \\
\text { frequencies }(\mathrm{Hz})\end{array}$ & $\begin{array}{c}\text { Median } \\
\text { frequencies }(\mathrm{Hz})\end{array}$ & RMS (mV) \\
\hline 8 & 168.83 & 167.12 & 0.0839 \\
\hline 16 & 95.47 & 94.21 & 0.3886 \\
\hline 32 & 51.70 & 50.59 & 0.6281 \\
\hline 64 & 26.77 & 26.25 & 0.4095 \\
\hline 128 & 14.93 & 13.89 & 0.3265 \\
\hline
\end{tabular}

With both transfer functions, tan-sigmoid and log-sigmoid, parameters were set as follows:

1) Training algorithm: Levenberg-Marquardt

2) No. of epochs or iterations: 1000

3) Performance goal: 0.001 or $0.1 \%$ error

4) Maximum performance gradient: $1 \times 10^{-10}$

Using the two transfer functions, tan-sigmoid and log-sigmoid, and the neuron numbers in the hidden layer were set between 3 and 10, hence there were 16 different ANNs to be trained. After the training, the 16 ANNs went through the validation process using the untrained data from the rest of the ten subjects. The performance goal or the error results between the 16 ANNs were then compared.

\section{RESUlTS}

Table I shows a sample of extracted features from a subject who exerted $75 \%$ of the MVIC from his right leg's vastus lateralis at selected scales for scalogram.

After training the 35 sets of data and validating with the remaining set of ten data, the transfer function which met the network target vector was the tan-sigmoid transfer function with neuron numbers 4, 5, 6 and 7 in the hidden layer. None of the log-sigmoid transfer function networks met the target after validation. The tan-sigmoid ANNs with the mentioned neuron numbers produced more comparable results in terms of meeting the performance goal. ANNs with neuron numbers 3, 8, 9 and 10 did not meet the target with some number of epochs or iterations being too great compared to the ANNs with neuron numbers 4, 5, 6 and 7 .

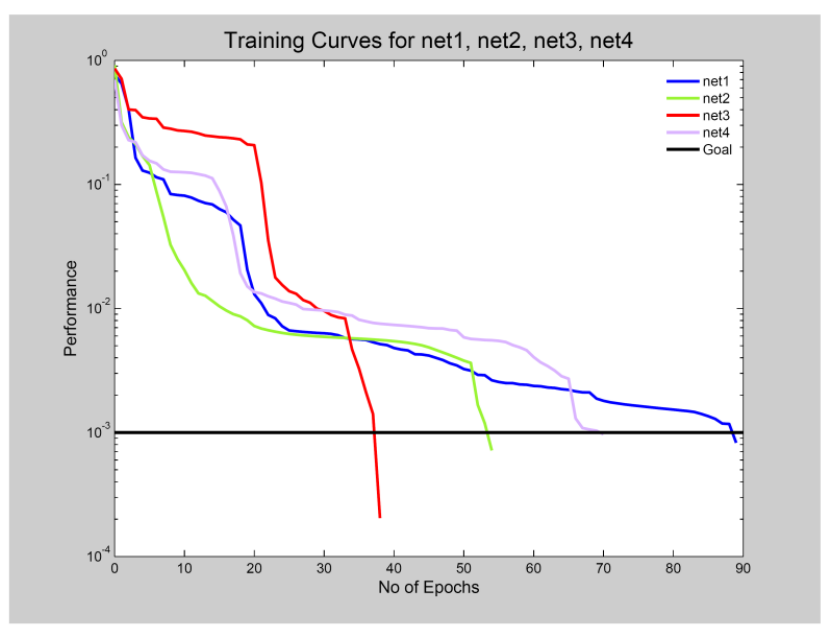

Fig. 7. The training of ANN net1, net 2, net 3 and net 4 showing efficiency in reaching the performance goal of 0.001 or $0.1 \%$ error 
TABLE II: SUMMARY OF RESULTS OF TRAINING AND VALIDATING NEURAL NETWORKS USING TAN-SIGMOID TRANSFER FUNCTION

\begin{tabular}{|c|c|c|c|}
\hline Network name & $\begin{array}{c}\text { Neuron number } \\
\text { at Hidden layer }\end{array}$ & Error & Error Percentages \\
\hline net1 & 4 & $6 / 30$ & 20 \\
\hline net2 & 5 & $2 / 30$ & 6.67 \\
\hline net3 & 6 & $1 / 30$ & 3.33 \\
\hline net4 & 7 & $7 / 30$ & 23.33 \\
\hline
\end{tabular}

The network with neuron number 4 in the hidden layer is named as 'net1', the one with neuron number 5 is 'net 2 ' and so on. Ten sets of untrained data from 10 subjects were validated, 15 input features were tested for three targets from each subject. With ten subjects, 10 validations with Boolean notation results were produced for each of the three output targets, which are $25 \%, 50 \%$ and $75 \%$ MVIC, totalling 30 validation results.

Fig. 7 shows the comparison between training curves of the different neuron numbers in the hidden layer with efficiency in reaching the performance goal. The summary of results of the ANNs which met the target is listed in Table II.

\section{DISCUSSION AND CONCLUSION}

Extracting feature from the whole 10-second signal would be an ideal as it may show more variants of the signal characteristics. Processing the whole duration at one time would require a vast amount of power from the computer and disk-space to run. Such facilities are not available at this stage. Breaking the signals into short time durations would be more achievable for feature extraction, but a laborious task. At this stage, processing the 2 -second region is sufficient to represent the signal characterisation. Researching into extracting the features from the whole signal duration could be expanded for the future work in this research.

After applying the CWT analysis to this 2-second quasi-stationary region, a scalogram was formed presenting the scale and time-based plot. This plot showed which scale at particular time was dominant indicated by the amplitude. The technique of scales to frequencies conversion is relatively new and found to be a powerful way of portraying scales. Scales are less familiar parameters, converting them to frequencies are making them a more common and meaningful parameters. This technique leads to the selection of the scale which would largely impact the whole signal characterisation.

Selecting the scale index numbers was carried out by visually viewing the scalogram and the frequency-time based spectrum plot. The dominant regions were noted with the corresponding scale numbers which were chosen. This technique required sound judgement and accuracy as it was done manually. Hence, the results can still be subjected by human error. Although, whichever index scale number is chosen, as long as the same ones are being used throughout the rest of the research process, the end results should give the same consistency. This is due to the nature of the values from any parameter being relative rather than absolute. Future work to develop an extension programming code for selecting dominant scale index numbers can be done to eliminate or minimise error.

So far, the ANN stage has produced satisfactory results with minimal error in terms of classifying an untrained normal muscle data as 'normal'. This showed that the basic procedure developed using ANN can be further utilised and refined for future work by collecting more data and investigating into setting features in different units. This technique is concluded to be a strong base method for further study in this research of developing muscle signal classifier.

Future work of data collection from abnormal muscles can be carried out where they can be used to test and validate the existing classifier which was built to classify for normal muscle. This technique can be executed for validating various classifiers that can be built for different muscles condition. It will need a vast amount of database of muscle signal for building this type of muscle condition diagnostic tool.

In summary, this research has investigated aspects involved in the digital signal processing using Continuous wavelet and Fourier analysis for extracting features of SEMG signals from 45 subjects with normal leg muscles. Then the use of ANN for developing a signal classifier was also explored. As an early stage in this area of research, various aspects were developed more in an outlined manner rather than a strong focus in one area using techniques from the literature review. More focus work on a particular area can be done in future work of this research.

\section{ACKNOWLEDGMENT}

This work was supported in part by the AUT University School of Engineering Research Grant with AUT Ethics Approval.

\section{REFERENCES}

[1] K. Englehart et al., "Classification of the Myoelectric Signal using Time-Frequency Based Representations," Medical Engineering \& Physics, pp. 431-438, 1999.

[2] R. Constable and R. J. Thornhill, "Using the Discrete Wavelet Transform For Time-Frequency Analysis of the Surface EMG Signal," ISA, vol. 16, pp. 121-127, 1993.

[3] P. J. Sparto et al., "Wavelet Analysis of Electromyography for Back Muscle Fatigue Detection During Dynamic Constant-Torque Exertions," ISA, vol. 33, pp. 82-87, 1997.

[4] M. Misiti et al., Wavelet Toolbox For Use with MATLAB. Natick, MA The MathWorks Inc., 2000.

[5] J. V. d. Vegte, Fundamentals of Digital Signal Processing: Prentice Hall, 2002.

[6] M. T. Young et al., "Biosignal Processing," in Introduction to Biomedical Engineering, J. D. Enderle, et al., Eds., ed San Diego, CA: Academic Press, 2000, pp. 233-278.

[7] J. A. Crowe et al., "Wavelet Transforms as a Potential Tool for ECG Analysis and Compression," J Biomed Eng, vol. 14, pp. 268-272, 1992

[8] R. Merletti and P. A. Parker, "Electromyography," Hoboken, New Jersey: John Wiley \& Sons, 2004.

[9] B. Kieft. (2005, 2 March). A Brief History of Wavelets. [Online] Available:

http://www.gvsu.edu/math/student_work/Kieft/Wavelets\%20-\%20Ma in\%20Main\%20Page.html

[10] G. Drost et al., "Motor Unit Characteristics in Healthy Subjects and Those with Postpoliomyelitis Syndrome: A High Density Surface EMG Study," Muscle Nerve, vol. 30, pp. 269-276, September 2004.

[11] R. O. Duda and P. E. Hart, Pattern Classification and Scene Analysis, New York: Wiley, 1973.

[12] J. T. Tou and R. C. Gonzalez, Pattern Recognition Principles. Reading, MA: Addison-Wesley, 1974.

[13] K. S. Fu, Syntactic Pattern Recognition and Applications, Englewood Cliffs, NJ: Prentice-Hall, 1982. 
[14] F. Rosenblatt, "The perceptron: A probabilistic model for information storage and organization in the brain," Psycho Rev, vol. 65, pp. 386-408, 1958.

[15] B. Widrow and M. A. Lehr, "30 years of adaptive neural networks: Perceptron, madaline and backpropagation," in Proc. IEEE, vol. 78, pp. 1415-1442, 1990.

[16] D. R. Hush and B. G. Horne, "Progress in supervised neural networks: What's new since Lippmann," IEEE Sig Process Mag. 8-39, 1993.

[17] K. Englehart et al., "Classification of the Myoelectric Signal using Time-Frequency Based Representations," Medical Engineering \& Physics, 1999.

[18] Surface Electromyography for Noninvasive Assessment of Muscle (SENIAM). (2005). Recommendations for sensor locations in hip or upper leg muscles - vastus medialis. [Online]. Available: http://www.seniam.org/quadricepsfemorisvastusmedialis.html.

[19] R. Merletti and L. R. Lo Conte, "Surface EMG Signal Processing During Isometric Contractions," J Electromyogr Kinesiol, vol. 7, pp. 241-250, 1997.

[20] A. Luttmann et al., "Electromyographical study on surgeons in urology - II. Determination of muscular fatigue," Ergonomics, vol. 39, pp. 298-313, 1996

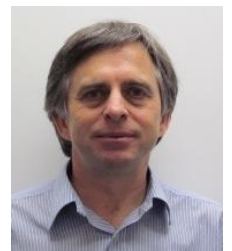

Jeff Kilby was born Edmonton, Alberta, Canada, has a MEng (Hons) in Signal Processing from the AUT University, Auckland New Zealand. Senior Lecturer in Electronics and Computing in the School of Engineering at AUT University.Main research topic is in the field of Biomedical Signal Processing and Devices with other research interests are LabVIEW Applications Micro-controller Applications and Wireless Sensor Network Applications

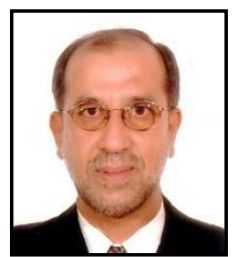

Krishnamachar Prasad has Ph.D. (University of Western Australia), M.Tech. (IIT Madras, India) and BE (Bangalore University, India) degrees, all in Electrical Engineering. Professor in the School of Engineering ay AUT University. His research focuses on the development and reliability studies of novel interconnects in conventional and three-dimensional integrated circuits. 